\title{
Entendendo as dimensões da Escala de Avaliação da Impulsividade pelo modelo CGF
}

\author{
Nelson Hauck Filho' \\ Universidade São Francisco, SP, Brasil \\ Fabián J. M. Rueda \\ Universidade São Francisco, SP, Brasil \\ Kelly de Bortoli Pisoni \\ Universidade São Francisco, SP, Brasil
}

\begin{abstract}
Resumo: O presente trabalho teve como objetivo verificar a contribuição dos Cinco Grandes Fatores (CGF) da personalidade na explicação dos domínios da Escala de Avaliação da Impulsividade (EsAvl). Participaram da pesquisa 335 servidores públicos do estado da Bahia, com idades entre 21 e 62 anos. Por meio de um modelo de path analysis, verificou-se que o fator falta de concentração e de persistência da EsAvl foi explicado por baixa conscienciosidade e alto neuroticismo, enquanto o fator controle cognitivo foi explicado por alta conscienciosidade e alta socialização, e o fator audácia/ temeridade se revelou uma combinação de reduzida socialização, reduzido neuroticismo e elevada abertura. Em contraste, os CGF explicaram apenas $1 \%$ do fator planejamento futuro. Os resultados proporcionam um entendimento aprofundado dos processos subjacentes aos domínios da EsAvl, revelando também de que maneira os fatores do instrumento se conectam aos CGF e a um proeminente modelo geral de impulsividade.
\end{abstract}

Palavras-chave: personalidade; impulsividade; path analysis; validade; avaliação psicológica.

MAPPING THE DIMENSIONS FROM THE IMPULSIVITY ASSESSMENT SCALE ONTO THE BIG FIVE

\begin{abstract}
In the present study, we investigated the contribution of the Big Five factors of personality in explaining the domains from the Impulsivity Assessment Scale (EsAvl). Participants included in the study were 335 public workers from the Brazilian State of Bahia, ages ranging from 21 to 62 years. Path analysis modeling revealed that lack of concentration was mainly predicted by low conscientiousness and high neuroticism, whereas cognitive control is related to high conscientiousness and high agreeableness, and what explains fearlessness is low agreeableness, low neuroticism, and high openness. In contrast, the Big Five factors accounted for only $1 \%$ of the variance in the planning scale. Findings provide an in-depth understanding of the processes underlying each EsAvl scale, showing how they connect to the Big Five factors of personality and a general model of impulsivity.
\end{abstract}

Keywords: personality; impulsivity; path analysis; validity; psychological assessment.

${ }^{1}$ Endereço de correspondência: Nelson Hauck Filho: R. Waldemar César da Silveira, 105, VI. Cura D'Ars (SWIFT), Campinas, SP. CEP: 13045-510. E-mail: hauck.nf@gmail.com 
ENTENDIENDO LAS DIMENSIONES DE LA ESCALA DE EVALUACIÓN DE LA IMPULSIVIDAD POR EL MODELO CGF

\begin{abstract}
Resumen: Este trabajo tuvo como objetivo verificar la contribución de los Cinco Grandes Factores (CGF) de la personalidad en la explicación de los dominios de la Escala de Evaluación de la Impulsividad (EsAvl). Participaron de la investigación 355 funcionarios públicos del Estado de Bahía, con edad entre 21 y 62 años. Por el modelo de path analysis se verifico que el factor falta de concentración y de persistencia de la EsAvl fue explicado por baja responsabilidad y alta inestabilidad emocional, mientras que el factor control cognitivo fue explicado por responsabilidad y sociabilidad altas; $y$ el factor audacia y temeridad se reveló una combinación de sociabilidad e inestabilidad emocional bajas y apertura a experiencias alta. Por su vez, los CGF explicaron apenas I\% del factor planeamiento futuro. Los resultados permitieron entender en profundidad los procesos subyacentes a los dominios de la EsAvl, mostrando también de qué forma los factores del instrumento se relacionan a los CGF y a un prominente modelo general de la impulsividad.
\end{abstract}

Palabras clave: personalidad; impulsividad; path analysis; validez; evaluación psicológica.

\title{
Introdução
}

A impulsividade envolve reduzida capacidade de planejamento, problemas atencionais e comportamentos de risco (Berg, Latzman, Bliwise, \& Lilienfeld, 2015). Do ponto de vista neuropsicológico, essa variável está relacionada à desvalorização pelo atraso (isto é, preferir recompensas imediatas, mesmo que menores - da Matta, Gonçalves \& Bizarro, 2012), a déficits na esquiva passiva (isto é, não aprender a inibir, seletivamente, alguns comportamentos - Newman, Widom, \& Nathan, 1985) e a falhas na modulação de respostas ou "desinibição" (isto é, problemas em ajustar o comportamento às demandas ambientais - Patterson \& Newman, 1993). A impulsividade desempenha papel central em diversas condições psicopatológicas, incluindo transtornos de humor (Najt, Perez, Sanches, Peluso, Glahn, \& Soares, 2007), transtornos da personalidade (Magyar, Edens, Lilienfeld, Douglas, \& Poythress, 2011) e transtornos relacionados ao desenvolvimento (Frick \& Viding, 2009). Não obstante, esse é um fenômeno multidimensional, cujas dimensões específicas podem envolver processos psicológicos latentes distintos. O presente estudo busca entender melhor cada domínio de um inventário de autorrelato de traços de impulsividade, a Escala de Avaliação da Impulsividade (EsAvl Ávila-Batista \& Rueda, 2013), tendo como base um modelo geral da personalidade, os Cinco Grandes Fatores (CGF).

O modelo dos CGF - Extroversão (E), Socialização (S), Conscienciosidade (C), Neuroticismo (N) e Abertura (A) - tem sido empregado como plano conceitual para o entendimento de diversas psicopatologias relacionadas à impulsividade. Em um estudo de metanálise de O'Boyle, Forsyth, Banks, Story, e White (2014), por exemplo, os CGF foram capazes de explicar $88 \%$ da variância em inventários avaliativos de traços de psicopatia. O perfil prototípico da psicopatia se mostrou uma combinação de reduzidos escores nos fatores $S(r=-0,42)$ e $C(r=-0,31)$. Outro estudo de metanálise revelou um perfil, a partir dos CGF, para os dez transtornos da personalidade definidos pelo DSM-IV-TR (Samuel \& Widiger, 2008). Os dois transtornos mais relacionados à impulsividade, borderline e antissocial, apresentaram, ambos, relações negativas com os fatores 
$S\left(r_{\text {antissocial }}=-0,36, r_{\text {borderline }}=-0,24\right)$ e $C\left(r_{\text {antissocial }}=-0,33, r_{\text {borderline }}=-0,29\right)$, com o adicional de que o transtorno borderline também apresentou uma correlação positiva com o fator $\mathrm{N}(r=0,54)$. O uso de substâncias, outro aspecto típico da impulsividade, também pode ser mapeado nos CGF. A metanálise de Malouff, Thorsteinsson, Rooke, e Schutte (2007), por exemplo, relacionou o uso de álcool a escores nos fatores $C$ $(r=-0,22), \mathrm{S}(r=-0,17)$ e $\mathrm{N}(r=0,15)$. Uma interpretação geral desses resultados é que aspectos impulsivos parecem envolver uma tendência à experiência de afetos negativos $(\mathrm{N}+)$, uma reduzida capacidade de organização e de engajamento em tarefas cognitivas (C-) e uma tendência ao antagonismo e à não cooperação (S-). Provavelmente, essa configuração complexa reflete o fato de que comportamentos impulsivos possuem mais de um curso desenvolvimental possível (Newman \& Lorenz, 2009), e envolvem mais de uma dimensão temperamental (Patrick, Fowles, \& Krueger, 2009).

Um modelo compreensivo que busca explicitar essa diversidade de dimensões e de trajetórias desenvolvimentais da impulsividade é aquele proposto por Whiteside e Lynam (2001), a partir dos CGF. Os autores realizaram uma análise fatorial exploratória de 21 escalas (de nove diferentes instrumentos) avaliativas de aspectos da impulsividade, a fim de descobrir dimensões mais básicas dessa característica da personalidade. Foram encontradas quatro dimensões: premeditação (isto é, capacidade de postergar ações em favor de planejamento e de reflexão), urgência (isto é, descontrole quando sob afetos negativos), busca de sensações (isto é, necessidade de estímulos e de aventura) e falta de perseverança (isto é, dificuldades para continuar uma tarefa sem sentir tédio). Em termos dos CGF, a premeditação e a falta de perseveração envolveram, principalmente, reduzidos escores em $\mathrm{C}$ (o primeiro foi positivamente associado à deliberação, enquanto o segundo se associou negativamente à autodisciplina), urgência se associou a altos escores em $\mathrm{N}$, e busca de sensações esteve relacionada a altos escores em E. Esse modelo, que acumula um extenso rol de evidências empíricas a seu favor (Berg et al., 2015), ilustra como dimensões gerais do funcionamento da personalidade podem ser usadas como pano de fundo para o entendimento de fenômenos mais específicos, como a impulsividade.

No Brasil, recentemente, Ávila-Batista e Rueda (2013) desenvolveram a Escala de Avaliação da Impulsividade (EsAvl), um inventário de autorrelato composto por 31 itens, desenvolvido para captar aspectos gerais da impulsividade em adultos brasileiros. As análises exploratórias conduzidas sugeriram que o instrumento proporciona informação acerca de quatro fatores: falta de concentração e de persistência (isto é, déficits cognitivos e dificuldade em continuar tarefas; $\alpha=0,83$ ), controle cognitivo (isto é, preservado funcionamento executivo; $\alpha=0,84$ ), planejamento futuro (isto é, antecipação de consequências; $\alpha=0,75$ ) e audácia/temeridade (isto é, falha em apreciar riscos; $\alpha=0,56$ ). Uma investigação preliminar de Rueda, Ávila-Batista, e Pinto (2016) revelou relações coerentes entre os fatores encontrados para o instrumento e os domínios dos CGF. O fator falta de concentração e de persistência e o fator controle cognitivo se associaram mais a aspectos do fator C (relação negativa no primeiro caso, e positiva no segundo), apesar de também existir uma associação a características de 
$\mathrm{N}$ (relação positiva no primeiro caso, e negativa no segundo). A audácia/temeridade se associou positivamente a aspectos do fator $E$, e o fator planejamento futuro, por sua vez, não apresentou associação consistente com nenhum dos CGF. Nesse estudo, entretanto, os autores empregaram a Bateria Fatorial da Personalidade, instrumento que, embora seja uma das ferramentas mais utilizadas no Brasil na área, pode possuir conteúdo um pouco distinto quando comparado a instrumentos mais bem estabelecidos, como o NEO-PI (Costa \& McCrae, 1992).

O presente estudo busca aprofundar o entendimento dos fatores da EsAvl ao relacioná-los aos domínios dos CGF tal como avaliados pelo instrumento Inventário de Cinco Fatores NEO Revisado (NEO-FFI). O foco é investigar, em um modelo multivariado, a contribuição única de cada domínio dos CGF na explicação dos escores nos fatores da EsAVi. Essa estratégia é adequada no sentido de possibilitar um entendimento mais refinado do instrumento EsAvl e, potencialmente, uma comparação entre os fatores desse inventário e aquelas dimensões relatadas por Whiteside e Lynam (2001). As hipóteses aqui aventadas são que: 1) o fator falta de concentração e de persistência capta aspectos de reduzidos níveis em $C$, sendo próximo da dimensão falta de perseverança de Whiteside e Lynam (2001); 2) o fator controle cognitivo capta também aspectos de reduzidos níveis em $C$, sendo, no entanto, análogo à dimensão premeditação de Whiteside e Lynam; 3) o fator planejamento futuro envolve tanto elevados níveis em C quanto reduzidos níveis em $\mathrm{N}$, talvez captando características da dimensão premeditação e do polo inferior da urgência; e 4) o fator audácia/temeridade tem como base elevados níveis em $\mathrm{E}$, sendo análogo à dimensão busca de sensações. $O$ estudo não apenas consiste em uma investigação psicométrica acerca da EsAvl, mas também em um teste da flexibilidade do modelo de Whiteside e Lynam para o entendimento de instrumentos avaliativos de impulsividade em geral.

\section{Método}

\section{Participantes}

Participaram do estudo 335 adultos, com idades variando entre 21 e 62 anos ( $\mathrm{M}=$ 32,43 , DP $=6,04$ anos), sendo $63,60 \%$ homens (213). Os participantes eram servidores públicos de diversos cargos da polícia civil do estado da Bahia.

\section{Instrumentos}

Escala de Avaliação da Impulsividade - EsAvl (Rueda \& Ávila-Batista, 2013). A EsAvl é um instrumento de autorrelato de traços de impulsividade composto por 31 itens. Cada item é pontuado em uma escala Likert, que varia de $1=$ nunca a $5=$ sempre. A ferramenta avalia quatro dimensões da impulsividade: falta de concentração e de persistência (incapacidade de manutenção do foco do sujeito sem que haja dispersão, bem como em continuar algo que já tenha dado início), controle cognitivo (capacidade de refletir antes de agir ou responder a estímulos), planejamento futuro (capacidade de planejamento da pessoa sobre ações que tenham efeito futuro) e audácia/ 
temeridade (capacidade de avaliação de situações que envolvam riscos, além de busca por aventura). $O$ estudo original revelou coeficientes alfa de 0,87 (falta de concentração e de persistência), 0,86 (controle cognitivo), 0,78 (planejamento futuro) e 0,56 (audácia/temeridade). Apesar de a consistência interna da escala de audácia/temeridade ficar um pouco inferior ao desejável, esse valor diz respeito à amostra do estudo original, não refletindo, necessariamente, o funcionamento da escala em toda a população de indivíduos. Destaca-se, ainda, que os autores do estudo original decidiram manter os itens da escala por motivos de coesão teórica.

NEO-FFI (Costa \& McCrae, 1992; adaptado para o Brasil por Flores-Mendoza, 2010). O NEO FFI é composto por 60 itens pontuados em uma escala Likert, que varia de $1=$ discordo fortemente a 5 = concordo fortemente. $O$ inventário permite avaliar as cinco dimensões básicas da personalidade relatadas no Modelo dos Cinco Grandes Fatores: extroversão (E), socialização (S), conscienciosidade (C), neuroticismo (N) e abertura (A). Cada escala conta com 12 itens. No estudo de Flores-Mendoza (2010), análises de fidedignidade revelaram índices adequados de consistência interna $(0,70-0,83)$ e estabilidade teste-reteste $(0,77-0,90)$.

\section{Procedimentos de coleta de dados}

Inicialmente, o projeto de pesquisa teve a aprovação do Comitê de Ética em Pesquisa. Posteriormente, ocorreu a assinatura do Termo de Consentimento Livre e Esclarecido por todos os participantes do estudo. A aplicação dos instrumentos foi realizada de forma coletiva, em grupos de 20 pessoas, com duração aproximada de 50 minutos. A ordem de aplicação foi o instrumento EsAvl e então o NEO-FFI.

\section{Análise de dados}

Foram utilizados métodos descritivos para a caracterização da amostra, e métodos inferenciais foram empregados para investigar a relação entre os cinco grandes fatores de personalidade e os traços de impulsividade. Especificamente, foi testado um modelo de path analysis para investigar a contribuição de cada fator de personalidade na especificação dos fatores da EsAvl. Empregou-se o estimador maximum likelihood robusto (MLR) em função de possíveis desvios da normalidade nas variáveis do estudo. $\mathrm{O}$ ajuste do modelo foi avaliado a partir do teste qui-quadrado (que não deve ser significativo) e dos índices de ajuste Confirmatory Fit Index (CFI; que deve ser igual ou superior a 0,95), Tucker-Lewis Index (TLl; que deve ser igual ou superior a 0,95) e Root Mean Square Error of Approximation (RMSEA; que deve ser igual ou inferior a 0,08). Foram seguidas as recomendações descritas na literatura (Hu \& Bentler, 1999). Não obstante, ressalta-se que a path analysis não foi usada para testar um modelo teórico em particular, mas sim para estimar as contribuições parciais entre as variáveis em um contexto multivariado, ou seja, após o controle da influência mútua entre elas. A abordagem é semelhante ao uso de correlações parciais, mas simplifica o procedimento em função da elaboração de uma análise apenas. Isso permite conhecer melhor a rede 
nomológica de cada fator do instrumento, uma vez que revela suas associações únicas com as variáveis externas de interesse. As análises foram conduzidas com o software $\mathrm{R}$, pacotes Psych e Lavaan.

\section{Resultados}

Primeiramente, foram explorados os aspectos descritivos das variáveis do presente estudo. As informações encontradas são apresentadas na Tabela 1. Verifica-se que todas as variáveis apresentaram distribuição significativamente desviante da normalidade, ainda que com medidas de assimetria não muito expressivas. Esse resultado em particular sustenta o uso de um estimador robusto na testagem do modelo hipotetizado, como feito no presente estudo.

\section{Tabela I. Informações descritivas das variáveis do estudo}

\begin{tabular}{lccccc}
\hline & Média & Desvio-padrão & Assimetria & Curtose & $\begin{array}{c}\text { SW } \\
(p \text {-valor })\end{array}$ \\
\hline Extroversão & 33,69 & 4,99 & $-0,25$ & 0,15 & 0,032 \\
Socialização & 38,87 & 4,19 & $-0,37$ & $-0,04$ & $<0,00$ I \\
Concienciosidade & 42,34 & 3,88 & $-0,48$ & $-0,44$ & $<0,00$ I \\
Neuroticismo & 11,62 & 5,48 & 0,34 & 1,19 & $<0,00$ I \\
Abertura & 31,88 & 5,20 & $-0,41$ & 0,46 & 0,002 \\
Falta de concentração e de persistência & 20,34 & 5,24 & 0,23 & $-0,49$ & $<0,00$ I \\
Controle cognitivo & 34,38 & 3,63 & $-0,71$ & 2,27 & $<0,00$ I \\
Planejamento futuro & 16,77 & 3,26 & $-0,12$ & $-0,47$ & $<0,00$ I \\
Audácia e temeridade & 14,34 & 3,04 & 0,54 & 0,46 & $<0,001$ \\
\hline
\end{tabular}

SW = Teste de normalidade Shapiro-Wilk.

Fonte: Elaborada pelos autores.

Na sequência, foram investigadas as relações lineares entre os fatores da EsAvl e os CGF. Conforme a Tabela 2, os padrões de associação se mostraram diversos de acordo com cada fator de impulsividade da escala EsAvl. Falta de concentração e de persistência apresentou um padrão de correlações negativas com E, S e C, e de correlações positivas com N e A. Enquanto isso, Controle cognitivo apresentou um padrão quase oposto (exceto pela correlação positiva, mas quase nula, com A). Planejamento futuro mostrou-se quase não relacionado aos CGF, e audácia/temeridade teve como correlação mais expressiva uma relação pequena e negativa com $\mathrm{S}$. 


\section{Tabela 2. Correlações bivariadas entre os fatores da EsAvl e os domínios do NEO-FFI}

\begin{tabular}{lcccc}
\hline & $\begin{array}{c}\text { Falta de concentração } \\
\text { e de persistência }\end{array}$ & Controle cognitivo & Planejamento & $\begin{array}{c}\text { Audácia/ } \\
\text { temeridade }\end{array}$ \\
\hline Extroversão & $-0,34^{*}$ & 0,16 & 0,05 & 0,15 \\
Socialização & $-0,30^{*}$ & $0,32^{*}$ & $-0,05$ & $-0,23^{*}$ \\
Concienciosidade & $-0,59^{*}$ & $0,49 *$ & 0,04 & $-0,01$ \\
Neuroticismo & $0,67^{*}$ & $-0,33$ & 0,05 & $-0,07$ \\
Abertura & 0,13 & 0,06 & 0,11 & 0,14 \\
\hline
\end{tabular}

$* p<0,05$.

Fonte: Elaborada pelos autores.

As relações bivariadas, ainda que informativas, não permitem detectar o relacionamento direto entre cada par de variáveis, uma vez que não são controladas as influências das demais variáveis em cada coeficiente. Por esse motivo, a etapa seguinte foi o teste do modelo de path analysis, que permite estimar todos os relacionamentos lineares, de maneira conjunta, em um mesmo modelo. Tendo em vista a não ortogonalidade entre as variáveis exógenas (os CGF), foram permitidas correlações entre elas, a fim de obter estimativas mais consistentes dos coeficientes de regressão. Em primeiro lugar, testou-se um modelo saturado, em que todos os CGF foram especificados como preditores dos fatores de impulsividade da EsAvl. Na sequência, fixaram-se em zero todos os coeficientes de regressão não significativos (modelo restrito), o que permitiu tornar os graus de liberdade $>1$ e, assim, usar o teste qui-quadrado e os índices de ajuste para avaliar o ajuste do modelo.

O modelo restrito, com os coeficientes não significativos fixados em 0 , obteve um excelente ajuste aos dados, $\chi^{2}(7)=5,18, p=0,638, \mathrm{CFI}=1,000, \mathrm{TLI}=1,015$, RMSEA $=$ 0,000 (IC 90\% 0,000-0,053). Os coeficientes padronizados encontrados para esse modelo são apresentados na Figura 1. A capacidade explicativa dos CGF no que diz respeito às quatro dimensões da EsAvl variou de $1 \%$ para Planejamento Futuro a $52 \%$ para Falta de Concentração e de Persistência. O fator Falta de Concentração e de Persistência se mostrou explicado, principalmente, por uma combinação de Conscienciosidade (-) e Neuroticismo (+), enquanto Controle Cognitivo foi explicado por Conscienciosidade (+) e Socialização (+), Planejamento Futuro por Abertura (+), e Audácia e Temeridade por Socialização (-), Neuroticismo (-) e Abertura (+). 
Figura I. Coeficientes estimados para o modelo final

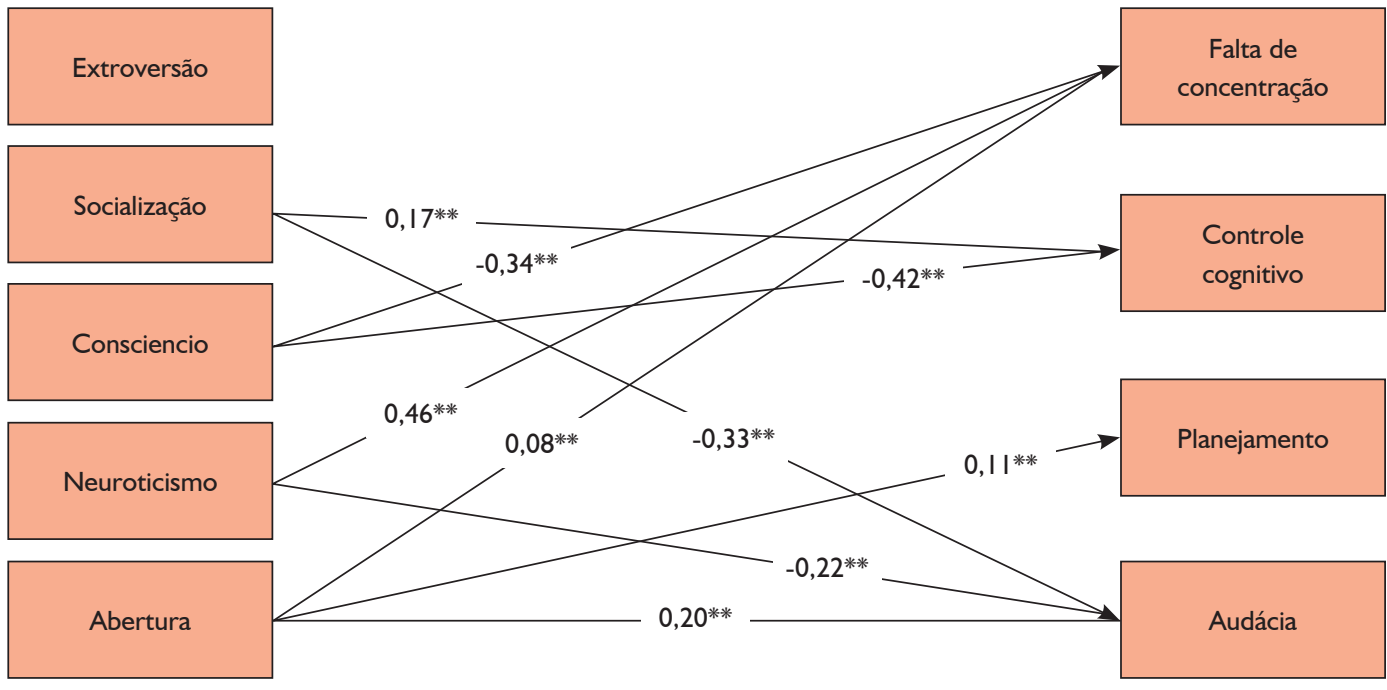

Nota: Variância explicada $\left(R^{2}\right)$ para os fatores da EsAvl: Falta de concentração e de persistência $=0,52$, Controle cognitivo $=$ 0,26 , Planejamento $=0,0 \mathrm{I}$, Audácia/temeridade $=0,12$. $* 0,05^{* *} p<0,0$ I.

Fonte: Elaborada pelos autores.

\section{Discussão e Conclusão}

O presente estudo teve como objetivo investigar a capacidade dos Cinco Grandes Fatores da personalidade de explicar as facetas da impulsividade tal como avaliadas pela EsAvl. Mais especificamente, o estudo propôs uma análise da possível configuração de traços de personalidade de cada um dos fatores da EsAvl em termo dos CGF do NEO-FFI. Os resultados sustentaram parcialmente as hipóteses aventadas, revelando um perfil de associações externas com os CGF que sustenta a diferenciação entre os fatores da EsAvl.

O fator falta de concentração e de persistência da EsAvl foi explicado por baixos escores em $\mathrm{C}$ e altos escores em $\mathrm{N}$. Por um lado, os achados confirmam a expectativa teórica de uma proximidade com a dimensão perseverança do modelo geral de Whiteside e Lynam (2001). Por outro lado, também sugerem uma sobreposição com a urgência, que é a única dimensão do modelo geral relacionada à impulsividade e também aquela mais associada à psicopatologia (Berg et al., 2015). Ou seja, essa dimensão da EsAvl parece reunir informação sobre os aspectos mais disfuncionais da impulsividade. Dada a relação negativa com $\mathrm{C}$ e positiva com $\mathrm{N}$, indivíduos que pontuam alto na dimensão estão mais propensos a serem desorganizados, desatentos e a terem problemas de regulação emocional, o que pode impactar, negativamente, no desempenho em diversos contextos sociais (por exemplo, Poropat, 2009). Vale ressaltar que esse padrão replica o que foi relatado por Rueda et al. (2016), cujo modelo de regressão identificou os mesmos fatores preditores, ainda que com outro instrumento de avaliação da personalidade, a Bateria Fatorial da Personalidade (BFP). 
O fator controle cognitivo da EsAvl foi explicado por altos níveis de C e S, perfil indicativo de um funcionamento adaptativo, ao contrário da escala de falta de concentração e de persistência. Esse resultado confirma a hipótese de uma proximidade com a dimensão premeditação do modelo geral de impulsividade proposto por Whiteside e Lynam (2001), mas também sugere a presença de um elemento de consideração empática pelos outros. Ou seja, a combinação de altos escores em C e S sugere que indivíduos que pontuam alto no fator controle cognitivo planejam melhor o curso de suas ações, inibindo respostas impulsivas e apresentando comportamentos mais ajustados às demandas sociais. Esse resultado replica apenas em parte o estudo de Rueda et al. (2016), que identificou como preditores da escala os fatores C (+), N (-) e $\mathrm{E}(-)$. Vale mencionar que a presença do fator $\mathrm{N}$ naquele estudo talvez se deva ao foco excessivo do fator $\mathrm{N}$ da BFP em aspectos antissociais, tradicionalmente mais próximos da dimensão S (cf. O'Boyle et al., 2014). Ainda assim, o principal preditor foi $\mathrm{C}$ em ambos os estudos (beta 0,42 no presente estudo, e 0,59 no estudo de Rueda et al., 2016). A escala, dessa forma, pode oferecer informação sobre recursos positivos que são essenciais às interações em diversos contextos.

Ao contrário do que foi hipotetizado, o fator planejamento futuro foi pouco explicado pelos domínios dos CGF, evidenciando apenas relação fraca e positiva com a dimensão A. Rueda et al. (2016) encontraram um resultado similar de fracas relações com os fatores da BFP, ainda que com uma relação pequena e positiva com $S(r=0,17)$. Assim, as evidências sugerem que o fator planejamento futuro contempla aspectos pouco avaliados pelos inventários tradicionais de traços de personalidade. Em vez de sugerir uma limitação do instrumento, é possível que essa escala proporcione uma informação clínica valiosa indisponível em outros instrumentos tipicamente usados para avaliar impulsividade. Novos estudos poderão investigar de que maneira escores em planejamento futuro se relacionam a desfechos na vida das pessoas (por exemplo, sucesso profissional e acadêmico), e, importante, se essa dimensão apresenta validade incremental na predição desses desfechos quando comparada às demais dimensões da EsAvl.

Por fim, a dimensão audácia e temeridade da EsAvl foi explicada por S (-), N (-) e A (+). Os achados do presente estudo rejeitam a hipótese simples de que essa dimensão refletiria, primariamente, altos escores em E. A despeito da similaridade de conteúdo com a dimensão busca de sensações do modelo de Whiteside e Lynam (2001), o padrão de associações parece sugerir que essa dimensão capta um domínio temperamental conhecido, precisamente, como "audácia" (boldness) (Patrick et al., 2009). Na literatura, a dimensão audácia do temperamento envolve um fenótipo destemido, reduzida ansiedade, dominância e assertividade. Na EsAvl, não obstante, a escala audácia e temeridade parece combinar esse componente temperamental a elementos de antagonismo e busca experiências, dada a associação negativa com N. Isso torna essa escala um possível marcador de alguns aspectos da personalidade psicopática, hipótese que necessitaria de atenção por parte de estudos futuros. De todo modo, altos 
escores na dimensão audácia e temeridade da EsAvl são sugestivos de disposição a novas experiências, falta de colaboração e reduzida responsividade ao perigo. Os achados contrariam as hipóteses de pesquisa e, parcialmente, os resultados encontrados por Rueda et al. (2016). O ponto essencial é que, em contraste com o presente estudo, a relação entre essa escala e o fator $\mathrm{N}$ foi positiva no estudo de Rueda et al. (2016). Essa diferença pode ter sido causada pelo fato (anteriormente mencionado) de que a escala N da BFP contém uma faceta de desajustamento psicossocial, que os autores relatam estar relacionada a aspectos antissociais (Nunes, Hutz, \& Nunes, 2010). Em outras palavras, o fator $\mathrm{N}$ da BFP contém elementos que são mais típicos de reduzido $\mathrm{S}$, o que explica as diferenças de associação encontradas.

O presente estudo tem como limitação o uso de dados coletados mediante procedimentos não aleatórios, o que pode impor restrições à generalização dos coeficientes encontrados ao considerar a população geral brasileira. Não obstante, os achados do presente estudo replicam em boa parte aqueles do estudo de Rueda et al. (2016), mesmo ao usar um instrumento diferente para avaliar os CGF. Vale mencionar também que a comparação da EsAvl com o modelo de Whiteside e Lynam (2001) teria se beneficiado da aplicação do instrumento desenvolvido por aqueles autores, o UPPS (Urgency, Premeditation, Perseverance and Sensation seeking). Todavia, o instrumento ainda não possui uma versão adaptada ao contexto nacional, sugestão que fica para novas investigações. Apesar dessas limitações, o estudo permitiu um entendimento aprofundado dos processos psicológicos avaliados pelas escalas da EsAvl. Foi possível não apenas efetuar um mapeamento das escalas da EsAvl no panorama dos CGF, mas também esboçar aproximações entre esses fatores e um modelo mais amplo de impulsividade.

\section{Referências}

Ávila-Batista, A. C., \& Rueda, F. J. M. (2011). Construção e estudos psicométricos de uma Escala de Avaliação da Impulsividade. Psico-USF, 16(3), 285-295. doi:10.1590/ S1413-82712011000300005

Ávila-Batista, A. C., \& Rueda, F. J. M. (2013). Escala de Avaliação da Impulsividade - Formas $A$ e $B$ (EsAvl-Formas $A$ e B). São Paulo: Vetor.

Berg, J. M., Latzman, R. D., Bliwise, N. G., \& Lilienfeld, S. O. (2015). Parsing the heterogeneity of impulsivity: A meta-analytic review of the behavioral implications of the UPPS for psychopathology. Psychological Assessment, 27(4), 11291146. doi:10.1037/pas0000111

Costa, P. T., \& McCrae, R. R. (1992). Revised NEO Personality Inventory (NEO PI-R) and NEO Five-Factor Inventory (NEO-FFI) professional manual. Odessa, FL: Psychological Assessment Resources. 
da Matta, A., Gonçalves, F. L., \& Bizarro, L. (2012). Delay discounting: Concepts and measures. Psychology \& Neuroscience, 5(2), 135-146. doi:10.3922/j.psns.2012.2.03

Flores-Mendoza, C. E. (2010). Estudo brasileiro do NEO-FFI-R (versão curta). São Paulo: Vetor.

Frick, P. J., \& Viding, E. (2009). Antisocial behavior from a developmental psychopathology perspective. Development and Psychopathology, 21(Special Issue 04), 1111-1131. doi:10.1017/S0954579409990071

Hu, L., \& Bentler, P. M. (1999). Cutoff criteria for fit indexes in covariance structure analysis: Conventional criteria versus new alternatives. Structural Equation Modeling: A Multidisciplinary Journal, 6(1), 1-55. doi:10.1080/10705519909540118

Magyar, M. S., Edens, J. F., Lilienfeld, S. O., Douglas, K. S., \& Poythress, N. G. (2011). Examining the relationship among substance abuse, negative emotionality and impulsivity across subtypes of antisocial and psychopathic substance abusers. Journal of Criminal Justice, 39(3), 232-237.

Malouff, J. M., Thorsteinsson, E. B., Rooke, S. E., \& Schutte, N. S. (2007). Alcohol involvement and the Five-Factor model of personality: a meta-analysis. Journal of Drug Education, 37(3), 277-294.

Najt, P., Perez, J., Sanches, M., Peluso, M. A. M., Glahn, D., \& Soares, J. C. (2007). Impulsivity and bipolar disorder. European Neuropsychopharmacology: The Journal of the European College of Neuropsychopharmacology, 17(5), 313-320. doi:10.1016/ j.euroneuro.2006.10.002

Newman, J. P., \& Lorenz, A. R. (2009). Response modulation and emotion processing: Implications for psychopathy and other dysregulatory psychopathology. In R. J. Davidson, K. L. Scherer, \& H. H. Goldsmith (Eds.), Handbook of Affective Sciences (pp. 904-929). New York: Oxford University Press, USA.

Newman, J. P., Widom, C. S., \& Nathan, S. (1985). Passive avoidance in syndromes of disinhibition: psychopathy and extraversion. Journal of Personality and Social Psychology, 48(5), 1316-1327.

Nunes, C. H. S. S., Hutz, C. S., \& Nunes, M. F. O. (2010). Bateria Fatorial de Personalidade (BFP): Manual técnico. São Paulo: Casa do Psicólogo.

O'Boyle, E. H., Forsyth, D. R., Banks, G. C., Story, P. A., \& White, C. D. (2014). A Meta-Analytic Test of Redundancy and Relative Importance of the Dark Triad and Five-Factor Model of Personality. Journal of Personality, n/a-n/a. doi:10.1111/jopy. 12126

Patrick, C. J., Fowles, D. C., \& Krueger, R. F. (2009). Triarchic conceptualization of psychopathy: developmental origins of disinhibition, boldness, and meanness. Development and Psychopathology, 21(3), 913-938. doi:10.1017/S0954579409000492 
Patterson, C. M., \& Newman, J. P. (1993). Reflectivity and learning from aversive events: toward a psychological mechanism for the syndromes of disinhibition. Psychological Review, 100(4), 716-736. Retrieved from http://www.ncbi.nlm.nih. gov/pubmed/8255955

Poropat, A. E. (2009). A meta-analysis of the five-factor model of personality and academic performance. Psychological Bulletin, 135(2), 322-338. doi:10.1037/a00 14996

Rueda, F. J. M., Ávila-Batista, A. C., \& Pinto, L. P. (2016). Impulsividad y facetas de la personalidad: relación entre instrumentos de medida. Ciencias Psicológicas (Uruguay), 10(1), 7-16.

Samuel, D. B., \& Widiger, T. A. (2008). A meta-analytic review of the relationships between the five-factor model and DSM-IV-TR personality disorders: a facet level analysis. Clinical Psychology Review, 28(8), 1326-1342. doi:10.1016/j.cpr.2008. 07.002

Whiteside, S. P., \& Lynam, D. R. (2001). The Five Factor Model and impulsivity: using a structural model of personality to understand impulsivity. Personality and Individual Differences, 30(4), 669-689. doi:10.1016/S0191-8869(00)00064-7

Submissão: 1.6.2016

Aceite: 1.11.2017 